\title{
ĐÁNH GIÁ ĐỘ CHÍNH XÁC CỦA ĐỊNH VỊ ĐIỂM ĐƠN SỦ DỤNG SỐ HIỆ CHỈNH THỜI GIAN THỤ̉C CỦA IGS
}

\author{
NGUYẼ̃N NGỌC LÂU ${ }^{(1)}$, PHẠM CẦN(2) \\ (I) Trường ĐH Bách Khoa TP. Hồ Chí Minh \\ ${ }^{(2)}$ Công ty TNHH Thiết bị Đại Nam
}

\section{Tóm tắt:}

Để đánh giá độ chính xác của định vị điểm đơn sử dụng số hiệu chỉnh thời gian thực tù dịch vu IGS thông qua mạng Internet tại Việt Nam. Chúng tôi đã xủ lý và đánh giá độ chính xác về các thành phần tọa độ của một trạm đo đặt tại TP. HCM vào ngày 08-11-2017. Kết quả cho thấy độ chính xác trung bình của định vị điểm đon sủ dụng RTS của các thành phần hướng Bắc, hương Đông và Độ cao tương ứng là $0.26(\mathrm{~m}), 0.21(\mathrm{~m})$ và 0.40 (m). Độ chính xác này cải thiện khoảng $75 \%$ về mặt bằng và $81 \%$ về độ cao so với khi định vị điểm đơn sủ dụng bản lịch truyền thông, và kém hơn khoảng 50\% so với kết quả hậu xủ lý dùng bản lịch chính xác và số hiệu chỉnh đồng hồ vệ tinh 30 giây của IGS $(0.11,0.10$ và $0.21 \mathrm{~m})$.

\section{Giới thiệu}

Định vị điểm đơn thời gian thực thông thường (dùng bản lịch truyền thông) chỉ đạt độ chính xác ở mức 5-15 (m) nên thường đáp ứng cho các ứng dụng yêu cầu độ chính xác thấp như: dẫn đường, định vị tàu thuyền trên biển,... Trong khi đó, kỹ thuật định vị điểm chính xác cao (Precise Point Positioning- PPP) hiện nay đã đạt được độ chính xác ở mức cm-dm, tuy nhiên PPP truyền thống chỉ phục vụ cho ứng dụng hậu xử lý vì phải cần đến các sản phẩm cuối cùng từ dịch vụ IGS với thời gian chờ đợi vài ngày.

Hiện nay dịch vụ thời gian thực của IGS (Real-time Service - RTS) cung cấp đến người dùng các sản phẩm ở thời gian thực bao gồm: số hiệu chỉnh về quỹ đạo và đồng hồ vệ tinh GPS. RTS dựa trên cơ sở mạng lưới các trạm IGS và các trung tâm phân tích dữ liệu để tính toán ra số hiệu chỉnh về quỹ đạo và đồng hồ vệ tinh có độ chính xác cao để cung cấp cho người dùng ngay ở thời gian thực thông qua mạng Internet $[9,12]$. Từ đó hình thành nên khái niệm định vị điểm đơn chính xác thời gian thực hay gần thời gian thực (Real Time Precise Point Positioning RTPPP).
RTPPP là phương pháp định vị điểm đơn, xử lý các trị đo pha và mã từ một máy thu GNSS duy nhất cùng với sử dụng các sản phẩm số hiệu chỉnh quỹ đạo và đồng hồ vệ tinh GNSS thời gian thực, chính xác từ Dịch vụ GNSS quốc tế (International GNSS Service - IGS) [5].

Trong bài báo trước đó [12], chúng tôi đã khảo sát độ chính xác của quỹ đạo và đồng hồ vệ tinh sau khi đã hiệu chỉnh bởi các số hiệu chỉnh thời gian thực của IGS thông qua việc so sánh với bản lịch IGS Final (đây là bản lịch có độ chính xác cao nhất theo thông báo của tổ chức IGS). Kết quả bài báo đó cho thấy rằng độ chính xác về quỹ đạo và đồng hồ vệ tinh GPS sau khi hiệu chỉnh bởi các số hiệu chỉnh RTS tương ứng khoảng $0.3 \mathrm{~m}$ và $1.2 \mathrm{~ns}$.

Trong bài báo này, chúng tôi sẽ trình bày nghiên cứu tiếp theo của chúng tôi về đánh giá độ chính xác định vị điểm đơn sử dụng số hiệu chỉnh thời gian thực của IGS. Trong đó chương trình định vị điểm được trình bày tóm tắt ở mục 2. Mục 3 giới thiệu về thiết bị thu và dữ liệu để khảo sát độ chính xác định vị RTPPP. Kết quả khảo sát và kết luận sẽ được trình bày ở mục 4 và 5 của bài báo.

Ngày nhận bài: 05/5/2018, ngày chuyển phản biện: 07/5/2018, ngày chấp nhận phản biện: 21/5/2018, ngày chấp nhận đăng: 25/5/2018 


\section{Quy trình xử lý}

2.1. Quy trình xủ̉ lý của chương trình RTPPP

Để thực hiện RTPPP, dữ liệu đầu vào cần có trị đo giả cự ly (trị đo mã) và trị đo pha trên 2 tần số, và bản lịch truyền thông, được xuất ra từ máy thu ở định dạng $\mathrm{RTCM}$ và đưa vào máy tính. Đồng thời máy tính thông qua kết nối Internet để nhận số hiệu chỉnh RTS để cải chính vào tọa độ và đồng hồ vệ tinh GPS, tính được từ bản lịch truyền thông. Trên cơ sở tọa độ và đồng hồ vệ tinh GPS đã được hiệu chỉnh bởi số hiệu chỉnh RTS cùng với trị đo nhận từ máy thu GPS đã được giải mã tiến hành tính toán ra tọa độ tại vị trí ăng-ten của máy thu. Sơ đồ khối của chương trình định vị điểm đơn sử dụng số hiệu chỉnh RTS được trình bày trong Hình 1 .

Trong đó:

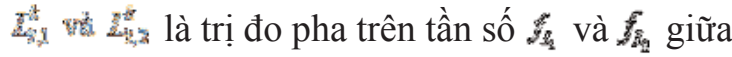
máy thu $i$ và vệ tinh $k$.

$2_{1}$ và $P_{i, 2}^{k}$ là trị đo mã trên tần số $\xi_{Z_{1}}$ và $F_{\delta_{2}}$ giữa máy thu $i$ và vệ tinh $k$.

$P_{i, 3}^{k}$ và $L_{i, 3}^{k}$ là trị đo mã và pha của tổ hợp không bị ảnh hưởng của điện ly giữa máy thu $i$ và vệ tinh $k$.

$X_{b}^{k}, \mathrm{Y}_{b}^{k}, Z_{b}^{k}, d I_{b}^{k}$ là tọa độ và đồng hồ vệ tinh $k$ khi tính toán từ bản lịch truyền thông.

$\partial X^{k}, \partial Y^{k}, \partial Z^{k}, \partial d T^{k}$ là số hiệu chỉnh quỹ đạo và đồng hồ vệ tinh từ RTS.

$X^{k}, \mathrm{Y}^{k}, Z^{k}, d T^{k}$ là tọa độ và đồng hồ vệ tinh $k$ sau khi đã hiệu chỉnh.

$A, P, C_{x}, L$ là các ma trận hệ số, trọng số và vector số hạng tự do.

$b_{i}^{k}$ là tham số đa trị (số thực).

$\delta X_{r}, \delta Y_{r}, \delta Z_{r}$ là số hiệu chỉnh cho ẩn số tọa độ máy thu.

$X_{r}, Y_{r}, Z_{r}$ là tọa độ của máy thu.

\subsection{Biện pháp khắc phục các nguồn sai số}

Ngoài các nguồn sai số do vệ tinh gây ra là sai số quỹ đạo và đồng hồ vệ tinh GPS, độ chính xác định vị điểm đơn còn chịu tác động của các nguồn sai số liên quan đến trị đo và phần cứng của máy thu [3]. Vì vậy, các nguồn sai số này cần được khử hoặc giảm đến mức tối đa khỏi phương trình trị đo nhằm cải thiện độ chính xác RTPPP.

- Các nguồn sai số liên quan đến trị đo GPS bao gồm:

+ Ảnh hưởng của sai số điện ly: dùng trị đo kết hợp không bị ảnh hưởng điện ly $\mathrm{P} 3$ và $\mathrm{L} 3$ để khử khỏi phương trình trị đo.

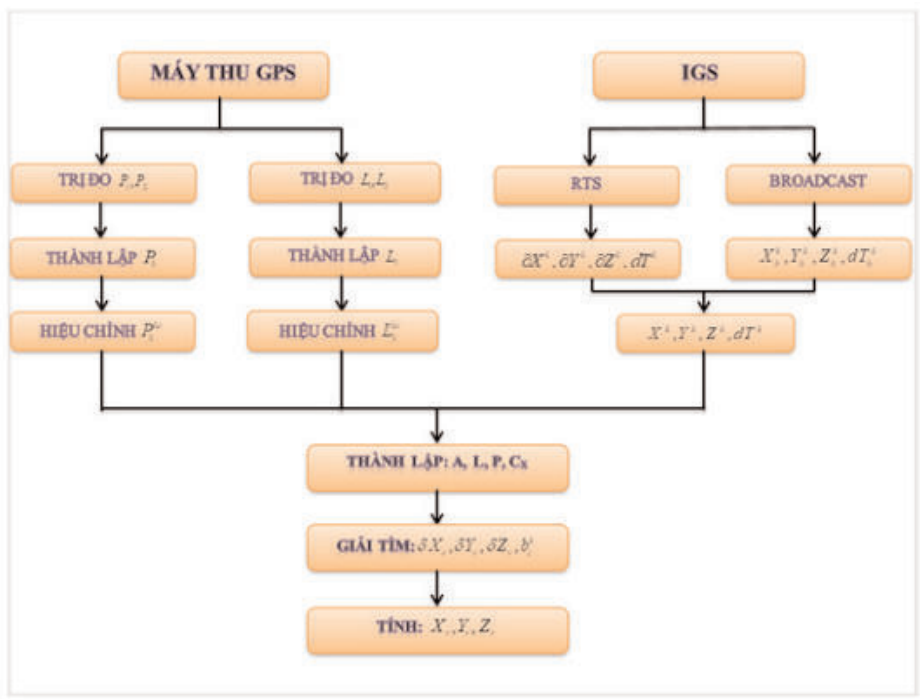

Hình 1: So đồ khối của chưong trình RTPPP sủ dụng số hiệu chỉnh RTS 
+ Ảnh hưởng của sai số đối lưu: dùng mô hình khí quyển chuẩn và hàm ánh xạ đối lưu để tính toán ra độ trễ và hiệu chỉnh vào trị đo.

+ Số hiệu chỉnh phase wind-up (chỉ ảnh hưởng đến trị đo pha): tính toán và hiệu chỉnh vào trị đo pha trong định vị điểm chính xác.

+ Tham số đa trị trong trị đo pha: được xem như các ẩn số thực trong phương trình trị đo pha.

- Các nguồn sai số liên quan đến thiết bị thu GPS bao gồm:

+ Sai số đồng hồ máy thu: lấy hiệu trị đo giữa các vệ tinh GPS sẽ khử hoàn toàn nguồn sai số này.

+ Sai số do độ trễ phần cứng và đa đường: chúng tôi bỏ qua và không xem xét đến.

+ Sai số do hiện tượng triều: nguồn sai số này ảnh hưởng chủ yếu vào thành phần độ cao. Trong kết quả nghiên cứu của bài báo cũng bỏ qua ảnh hưởng của nguồn sai số này.

\section{Thiết bị thu GPS và tập dữ liệu thực nghiệm}

Để thực hiện RTPPP sử dụng số hiệu chỉnh thời gian thực IGS-RTS, chúng tôi sử dụng máy thu 2 tần số Topcon Legacy-E đặt tại mốc DNE01 thu thập dữ liệu liên tục trong 1 ngày. Máy thu được kết nối với máy tính để nhận số liệu thời gian thực gồm: trị đo giả cự ly và trị đo pha của vệ tinh GPS theo định dạng RTCM xuất ra từ máy thu. Đồng thời, máy tính cũng nhận được số hiệu chỉnh RTS và bản lịch truyền thông từ dịch vụ IGS thông qua mạng Internet.

Để phục vụ cho việc đánh giá độ chính xác RTPPP, tọa độ chính xác của trạm đo dùng làm cơ sở để so sánh được xác định bằng phương pháp hậu xử lý PPP tĩnh, được cung cấp từ dịch vụ xử lý GPS online CSRS-PPP với độ chính xác chỉ ở mức mm-cm $[2,3,4]$. (Xem hình 2)

\section{Kết quả xử lý và phân tích độ chính xác}

Dữ liệu trị đo được thu vào ngày 08-11-2017 cùng với bản lịch truyền thông và số hiệu chỉnh quỹ đạo và đồng hồ vệ tinh GPS của RTS-IGS. Dữ liệu này được thực hiện bằng chương trình xử lý và đánh giá độ chính xác RTPPP do chúng tôi viết ra bằng ngôn ngữ lập trình MATLAB. Một số cài đặt chung cho quá trình xử lý được cho ở Bảng 1.

Dữ liệu của trạm đo được xử lý theo 4 phương án:

- Phương án 1: dùng bản lịch truyền thông.

- Phương án 2: dùng bản lịch truyền thông kết hợp với số hiệu chỉnh RTS.

- Phương án 3: Dùng bản lịch chính xác IGS Final và số hiệu chỉnh đồng hồ vệ tinh 15 phút

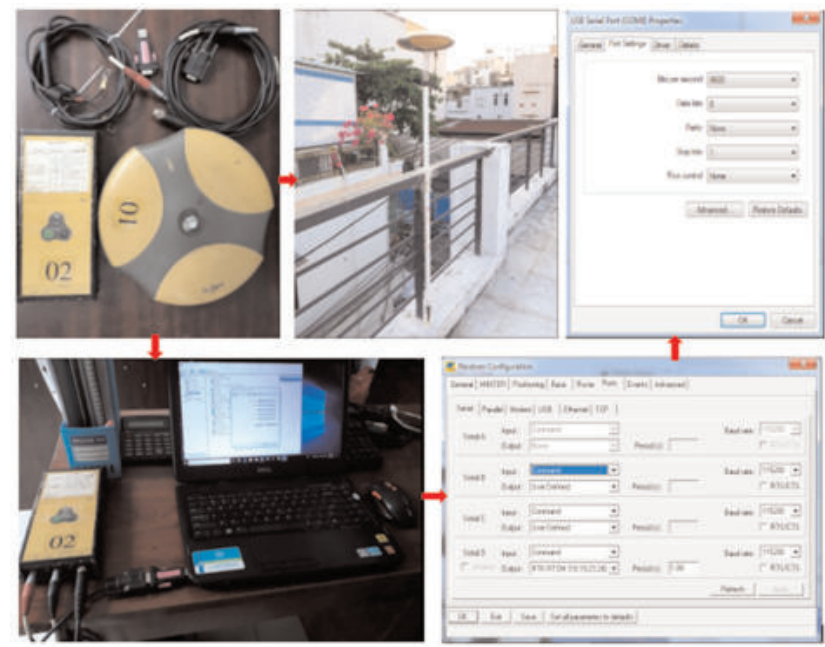

Hình 2: Cài đặt ăng-ten tại mốc DNE01 và kết nối máy thu Topcon Legacy-E với máy tính laptop 
(có tần suất cập nhật 15 phút/lần).

- Phương án 4: Dùng bản lịch chính xác IGS Final và số hiệu chỉnh đồng hồ vệ tinh 30 giây (có tần suất cập nhật 30 giây/lần).

Bảng 1: Các tham số cài đặt trong xủ lý RTPPP

\begin{tabular}{|c|c|}
\hline Nội dung & Giá trị \\
\hline \begin{tabular}{|l}
$\begin{array}{l}\text { Bản lịch và đồng hồ } \\
\text { vệ tinh }\end{array}$ \\
\end{tabular} & $\begin{array}{l}\text { Dùng bản lịch truyền thông và } \\
\text { số hiệu chînh RTS }\end{array}$ \\
\hline Trị đo & $\begin{array}{l}\text { Trị đo mã P3 và pha } \Phi_{3} \text { của vệ } \\
\text { tinh GPS }\end{array}$ \\
\hline Trọng số trị đo & $\operatorname{Sin}(\varepsilon), \varepsilon$ là góc cao vệ tinh \\
\hline \multirow{3}{*}{ Độ trễ đối lưu } & $\begin{array}{l}\text { Mô hình khí quyển chuẩn } \\
\text { UNB3m }\end{array}$ \\
\hline & 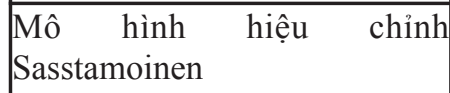 \\
\hline & Hàm ánh xạ Neill \\
\hline Giải đa trị & $\begin{array}{l}\text { Xem tham số đa trị là ẩn số } \\
\text { thực trong phương trình đo pha }\end{array}$ \\
\hline
\end{tabular}

Tọa độ trạm đo nhận được từ xử lý được so sánh với giá trị chính xác của nó theo 3 thành phần: hướng Bắc, hướng Đông và Độ cao theo công thức sau:

$$
\begin{aligned}
& m_{N}= \pm \sqrt{\frac{\sum_{i=1}^{n}\left(\mathrm{~N}_{i}-\mathrm{N}\right)^{2}}{n}} \\
& m_{E}= \pm \sqrt{\frac{\sum_{i=1}^{n}\left(\mathrm{E}_{i}-E\right)^{2}}{n}} \\
& m_{U}= \pm \sqrt{\frac{\sum_{i=1}^{n}\left(\mathrm{~h}_{i}-h\right)^{2}}{n}}
\end{aligned}
$$

Trong đó $\mathrm{N}_{\mathrm{i}}, \mathrm{E}_{\mathrm{i}}, \mathrm{h}_{\mathrm{i}}$ là tọa độ mặt bằng và độ cao của điểm DNE01 xác định được ở thời điểm thứ $\mathrm{i}$, còn $(\mathrm{N}, \mathrm{E}, \mathrm{h})$ là tọa độ và độ cao chính xác của điểm DNE01.

Kết quả so sánh khi xử lý từng thời điểm (epoch-by-epoch) cho ở Bảng 2.

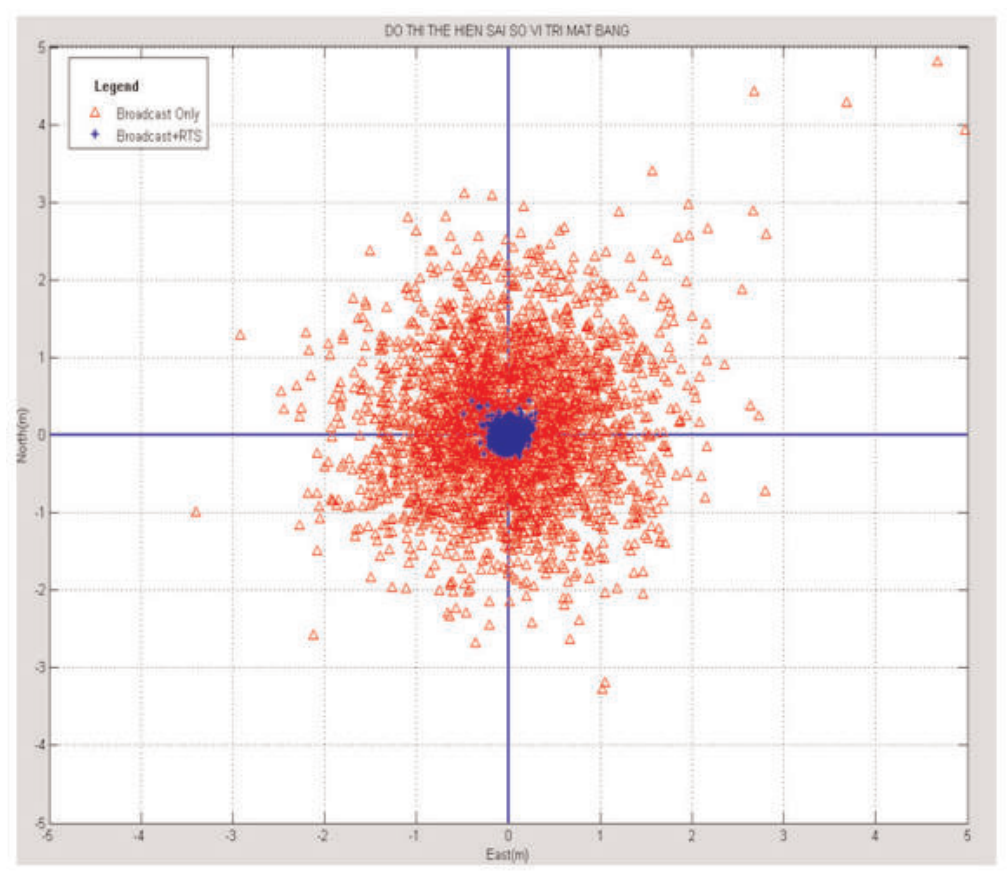

Hình 3: Đồ thị thể hiện độ lệch vị trí mặt bằng khi định vị có (dấu sao màu xanh) và không có sủ dụng số hiệu chỉnh RTS (tam giác màu đỏ) 
Đồ thị sai số vị trí mặt bằng khi định vị điểm đơn chỉ sử dụng bản lịch truyền thông (phương án 1) và sử dụng bản lịch truyền thông có hiệu chỉnh RTS (phương án 2) được biểu diễn theo đồ thị Hìn 3.

Theo Bảng 2, độ chính xác RTPPP sử dụng số hiệu chỉnh RTS đạt được là $0.21,0.26$ và 0.40 (m) theo hướng Đông, Bắc và Độ cao. So với kết quả định vị chỉ dùng bản lịch truyền thông, độ chính xác được cải thiện ở các thành phần: hướng Đông $(0.21 / 0.87$ 76\%), hướng Bắc $(0.26 / 1.01 \sim 74 \%)$ và Độ cao $(0.40 / 2.14 \sim 81 \%)$.

Trong Bảng 2 cũng cho thấy kết quả khi xử lý từng thời điểm (epoch-by-epoch) sử dụng số hiệu chỉnh RTS cho độ chính xác tương đương với kết quả dùng bản lịch chính xác SP3. Điều này cho thấy độ chính xác và tính ổn định của số hiệu chỉnh RTS về quỹ đạo và đồng hồ vệ tinh GPS là tương đối cao. Theo Hình 3, sai số về vị trí mặt bằng khi định vị điểm đơn dùng số hiệu chỉnh RTS ổn định và tập trung hơn so với định vị điểm đơn thông thường dùng bản lịch truyền thông.

Đồng thời theo Bảng 2, chúng ta thấy rằng độ chính xác của phương án 4 (có sử dụng số hiệu chỉnh đồng hồ vệ tinh 30 giây) ở các thành phần hướng Đông, Bắc và Độ cao là $(0.10,0.11,0.21)$ $\mathrm{m}$ tốt hơn phương án 3 (sử dụng số hiệu chỉnh đồng hồ vệ tinh 15 phút) là $(0.13,0.15,0.25) \mathrm{m}$. Điều này có thể giải thích rằng có sự sai lệch về kết quả nội suy cho số hiệu chỉnh đồng hồ vệ tinh GPS cho những thời điểm cách xa các thời điểm 15 phút chẵn.

Theo kết quả của các nghiên cứu của các tác giả trước đó [3], độ chính xác khi hậu xử lý Kinematic PPP có thể đạt ở mức dưới $\mathrm{dm}$. Các kết quả này cho độ chính xác tốt hơn kêt quả của chúng tôi khi xử lý bằng bản lịch IGS Final và số hiệu chỉnh đồng hồ vệ tinh 30 giây. Lý do của sự khác biệt này có thể kể đến là máy thu GPS và ăng-ten chúng tôi là loại thông thường, không tốt như các trạm IGS thường trực, nên không có khả năng giảm đa đường và loại nhiễu tốt như tại các trạm IGS, đồng thời điểm tham khảo ăng-ten được chúng tôi xác định tương đối bằng thước thép. Ngoài ra tọa độ cơ sở dùng để so sánh và đánh giá độ chính xác định vị có được thông qua việc gửi số liệu đến các dịch vụ xử lý GPS online chỉ đạt độ chính xác cm.

\section{Kết luận}

Chúng tôi đã khảo sát độ chính xác của định vị điểm đơn sử dụng số hiệu chỉnh RTS tại trạm đo DNE01 vào ngày 08-11-2017 bằng máy thu Topcon Legacy-E. Kết quả được tóm tắt như sau:

Bảng 2: So sánh kết quả xủ lý tùng thời điểm theo 4 trương hợp khảo sát

\begin{tabular}{|c|c|c|c|c|}
\hline STT & Phương án xử lý & East $(\mathbf{m})$ & North $(\mathbf{m})$ & Up $(\mathbf{m})$ \\
\hline 1 & Dùng bản lịch truyền thông & 0.872 & 1.005 & 2.137 \\
\hline 2 & Dùng bản lịch truyền thông + RTS & 0.213 & 0.260 & 0.398 \\
\hline 3 & $\begin{array}{c}\text { Dùng bản lịch IGS Final và số hiệu } \\
\text { chỉnh đồng hồ 15 phút }\end{array}$ & 0.126 & 0.146 & 0.252 \\
\hline 4 & $\begin{array}{c}\text { Dùng bản lịch IGS Final và số hiệu } \\
\text { chỉnh đồng hồ 30 giây }\end{array}$ & 0.103 & 0.111 & 0.213 \\
\hline
\end{tabular}


Độ chính xác khi xử lý từng epoch của RTPPP có thể đạt được $0.21,0.26$ và $0.40(\mathrm{~m})$ theo hướng Đông, hướng Bắc và Độ cao. So với khi xử lý chỉ sử dụng bản lịch truyền thông, độ chính xác này cải thiện đáng kể ở thành phần mặt bằng khoảng $75 \%$ và Độ cao khoảng $81 \%$.

Việc định vị điểm đơn dùng bản lịch chính xác IGS Final và số hiệu chỉnh đồng hồ vệ tinh 30 giây cho kết quả định vị có độ chính xác tốt nhất là $0.11(\mathrm{~m}), 0.10(\mathrm{~m})$ và $0.21(\mathrm{~m})$ ở các thành phần hướng Bắc, hướng Đông và Độ cao.

Với độ chính xác đạt được của RTPPP sử dụng số hiệu chỉnh RTS, cho phép chúng ta mở rộng mục đích ứng dụng so với định vị dùng bản lịch truyền thông. Chẳng hạn như phục vụ cho mục đích dẫn đường chính xác hay cho công tác đo vẽ địa hình đáy biển, sông, hồ và các ứng dụng yêu cầu độ chính xác trung bình. $\bigcirc$

\section{Tài liệu tham khảo}

[1]. Junbo Shi, Chaoquian Xu, Jiming Guo, Yang Gao, "A Performance Analysis of RealTime Precise Point Positioning for Deformation Monitorin," September 2013.

[2]. Nguyen Ngoc Lau, Tran Trong Duc, Duong Tuan Viet, Dang Van Cong Bang, "Automatic GPS precise point processing via internet", Report of ministry level project B2010-30-33, 2010.

[3]. Nguyen Ngoc Lau, Ho Chi Minh City University of Technology, "How Accuracy GPS Precise Point Positioning Can Be Achieved," Science \& Technology Development., Vol. 12, No. 08 - 2009.

[4]. Nguyen Ngoc Lau, Ho Chi Minh City University of Technology, "Point Precise
Positioning Using GPS and GLONASS Measurements," Journal of Geodesy and Cartography, No. 15 - March 2013.

[5]. Thomas Grinter, Craig Roberts, "Real Time Precise Point Positioning: Are We There Yet," International Global Navigation Satellite Systems Society, IGNSS Symposium 2013.

[6]. Junbo Shi, Chaoqian Xu, Jiming Guo, and Yang Gao, "Real-Time GPS Precise Point Positioning-Based Precipitable Water Vapor Estimation for Rainfall Monitoring and Forecasting," IEEE Transactions on Geoscience and RemoteSensing, June 2015.

[7]. Jan Kouba, Geodetic Survey Division Natural Resources Canada, "A GUIDE TO USING INTERNATIONAL GNSS SERVICE (IGS) PRODUCTS," Updated September 2015.

[8]. Andrea Sturze, Leos Mervart, Wolfgang Sohne, Georg Weber, Gerhard Wubbena, "RealTime PPP using open CORS Networks and RTCM Standards," 3rd International Conference on Machine Control \& Guidance, March 27-29, 2012.

[9]. Min-Wook Kang, Jihye Won, Mi-So Kim, Kwan-Dong Park, "Accuracy Evaluation of IGS-RTS Corrections to Stand-Alone Positioning Based on GPS Code-Pseudorange Measurements," Journal of Positioning Navigation, and Timing, 12-May-2016.

[10]. Tomoji Takasu, Tokyo University of Marine Science and Technology, "Real-time PPP with RTKLIB and IGS real-time satellite orbit and clock," IGS Workshop (2010).

[11]. Mohammed El-Diasty, Mohamed Elsobeiey, "Precise Point Positioning Technique 


\section{Nghiên cúu}

with IGS Real-Time Service (RTS) for Maritime Applications," Positioning, Nov-2015.

[12]. Nguyen Ngoc Lau, Pham Can, "Estimating the Precision of the IGS Real-Time GPS Satellites Orbit and Clock Corrections," The $15^{\text {th }}$ Conference on Science \& Technology, HCMUT, 20-Oct-2017.

[13]. M. El-Diasty, "Integrity Analysis of Real-time PPP Technique with IGS-RTS Service for Maritime Navigation," The International Archives of the Photogrammetry, Remote Sensing and Spatial Information Sciences, Volume XLII-4/W5, Kuala Lumpur, 04-Oct2017.

[14]. Hongzhou Yang, Yang Gao, "GPS Satellite Orbit Prediction at User End for RealTime PPP System," Journal of Sensors, Volume 17, 30-Aug-2017. $\mathrm{O}$

\section{Summary}

\section{Accuracy assessment of the precise point positioning using the IGS real-time corrections}

Nguyen Ngoc Lau

Ho Chi Minh City University of Technology

\section{Pham Can}

\section{Dainam Equipment Company Limited}

To assess the accuracy of single point positioning using IGS real time corrections via the Internet in Vietnam. We have processed and evaluated the accuracy of the coordinate components of a station located in Ho Chi Minh City on November 8th, 2017. The results show that the average accuracy of single point positioning using RTS of the north, east and up components is $0.26(\mathrm{~m}), 0.21$ (m) and $0.40(\mathrm{~m})$, respectively. This accuracy improves about $75 \%$ in horizontal and $81 \%$ in vertical components compared to single point positioning using broadcast ephemeride, and is about $50 \%$ less than post-processing results using the IGS Final precise ephemeride and 30s clock corrections $(0.11,0.10$ and $0.21 \mathrm{~m}) . \bigcirc$ 